\title{
Otology
}

\section{Expanded transcanal transpromontorial approach to the internal auditory canal and cerebellopontine angle: a cadaveric study}

\author{
Approccio allargato transcanalare transpromontoriale per il condotto uditivo \\ interno e l'angolo ponto-cerebellare: studio su cadavere
}

\begin{abstract}
L. PRESUTTI ${ }^{1}$, M. BONALI' ${ }^{1}$ D. MARCHIONI ${ }^{2}$, G. PAVESI ${ }^{3}$, A.FELETTI ${ }^{3}$, L. ANSCHUETZ ${ }^{14}$, M. ALICANDRI-CIUFELLI ${ }^{3}$ ${ }^{1}$ Otolaryngology-Head and Neck Surgery Department, University Hospital of Modena, Italy; ${ }^{2}$ Otolaryngology-Head and Neck Surgery Department, University Hospital of Verona, Italy; ${ }^{3}$ Neurosurgery Department, New Civil Hospital Sant'Agostino-Estense, Baggiovara (MO), Italy; ${ }^{4}$ Otolaryngology-Head and Neck Surgery Department, Inselspital, University Hospital and University of Bern, Switzerland
\end{abstract}

\begin{abstract}
SUMMARY
The aim of this paper is to describe and evaluate the feasibility of an expanded endoscopic transcanal transpromotorial approach (ExpTTA) to the internal auditory canal and the cerebellopontine angle. To this end, we performed a cadaveric dissection study in September 2015. In total, 2 heads ( 4 sides) were dissected focusing on anatomical landmarks and surgical feasibility. Data from dissections were reviewed and analysed for further consideration. In all 4 sides of the cadavers the procedure was feasible. In all cadavers, it was necessary to extensively drill the temporo-mandibular joint and to calibrate the external ear canal to allow adequate room to manoeuver the instruments and optics and to comfortably access the cerebellopontine angle. In addition, thorough skeletonisation of the carotid artery and the jugular bulb were necessary for the same purpose. In conclusion, ExpTTA appeared to be successful to access the internal auditory canal and cerebellopontine angle region. Potential extensive and routine application of this type of approach in lateral skull base surgery will depend on the development of technology and surgical refinements and on the diffusion of skull base endoscopic skills among otolaryngologists and neurosurgical community.
\end{abstract}

KEY WORDS: Cerebellopontine angle • Endoscopic ear surgery $\bullet$ Inner ear • Internal auditory canal $\bullet$ Transcanal approach

\section{RIASSUNTO}

Lo scopo dello studio è quello di descrivere e valutare la fattibilità di un approccio allargato transcanalare transpromontoriale al condotto uditivo interno e all'angolo pontocerebellare (ExpTTA). Nel settembre 2015 è stato condotto uno studio di dissezione su cadavere. In totale 2 teste (4 lati) son state dissecate focalizzando l'attenzione sull'anatomia chirurgica. I dati ottenuti dalle dissezioni son stati quindi analizzati. In tutti e quattro i lati è stato possibile eseguire la procedura, e tutti i punti di repere descritti son stati identificati. In tutti i cadaveri si è resa necessaria una ampia fresatura della articolazione temporo-mandibolare e il calibraggio del condotto uditivo esterno per permettere una adeguata esposizione e possibilità di manovra degli strumenti e le ottiche, e per accedere agevolmente all'angolo pontocerebellare. Anche la scheletrizzazione della carotide interna e del golfo della giugulare si sono rese necessarie con la stessa finalità. In conclusione l'ExpTTA si è dimostrata efficace per accedere chirugicamente al condotto uditivo interno e all'angolo pontocerebellare. Il potenziale uso estensivo e routinario di questo tipo di approccio alla pratica clinica dipenderà dallo sviluppo di tecnologie adeguate, dal rifinirsi di questa nuova tecnica, e dalla diffusione delle capacità manuali di chirurgia endoscopica del basicranio tra la comunità otorinolaringoiatrica e neurochirugica internazionale.

PAROLE CHIAVE: Angolo pontocerebellare $\bullet$ Chirurgia endoscopica dell'orecchio $\bullet$ Orecchio interno $\bullet$ Condotto uditivo interno $\bullet$ Approccio transcanalare

Acta Otorhinolaryngol Ital 2017;37:224-230

\section{Introduction}

Surgical approaches to the internal auditory canal (IAC) are widely known and extensively recorded, the most popular being classified as retrosigmoid, transmastoid-translabyrinthine and middle cranial fossa. The clinical indications, advantages, disadvantages and risks in terms of mortality and morbidity have been carefully described ${ }^{1}$.
A common factor in all of the methods described to date is that they are all indirect approaches to the inner ear, since the retrosigmoid and translabyrinthine methods approach the pathology posteriorly, while the middle cranial fossa approaches the pathology superiorly. To access the internal auditory canal (IAC) and cerebellopontine angle (CPA), all of these approaches require 
wide external incisions and a variable degree of bone removal ${ }^{1}$.

The first introduction of the endoscopic technique in IAC surgery was in combination with the retrosigmoid approach ${ }^{2}$. After removal of the CPA portion of the neoplasm, the intracanalicular extension was removed under endoscopic control, trying to avoid extensive drilling of the posterior aspect of the petrous bone. In surgical treatment of the middle ear, the endoscope was introduced in the $1990 \mathrm{~s}^{3}$ as an additive tool to visualise hidden areas ${ }^{4}$. During the last years, technical improvements and growing expertise in the handling of the endoscope allowed introducing an exclusive endoscopic approach to the middle ear ${ }^{56}$ and lateral skull base ${ }^{78}$. The development of these endoscopic techniques required several cadaver dissections ${ }^{9}$ to better understand the anatomy and to define appropriate instruments for this purpose. During these dissections, some advances were made in exploring the internal ear, from the labyrinth to the IAC, until an appropriate procedure was recorded, and ready to be applied clinically.
For the first time, an exclusive endoscopic approach to the IAC was described in and used to remove a cochlear schwannoma (CS) involving IAC in March 2012. The operation used a direct transcochlear approach from lateral to medial and from external to internal auditory canal, without any external incision ${ }^{10}$. Other lateral skull base applications were described during the last two years by our team ${ }^{78}$. The first case series of the exclusive endoscopic transcanal transpromontorial approach (EndoTTA) to remove vestibular schwannomas involving IAC has been recently published ${ }^{11}$.

The aim of this paper is to describe an expanded endoscopic transcanal transpromontorial approach (ExpTTA), derived from the EndoTTA, and to discuss the feasibility, results and findings of this approach on a cadaver model. Since management of intracanalicular vestibular schwannomas (VSs) is complex and strongly debated ${ }^{12}$, this kind of therapeutic option in appropriate and selected cases could modify classic concepts of the management of this pathology, even expanding the indications of EndoTTA, which at present are limited to small vestibular schwannomas of the IAC.

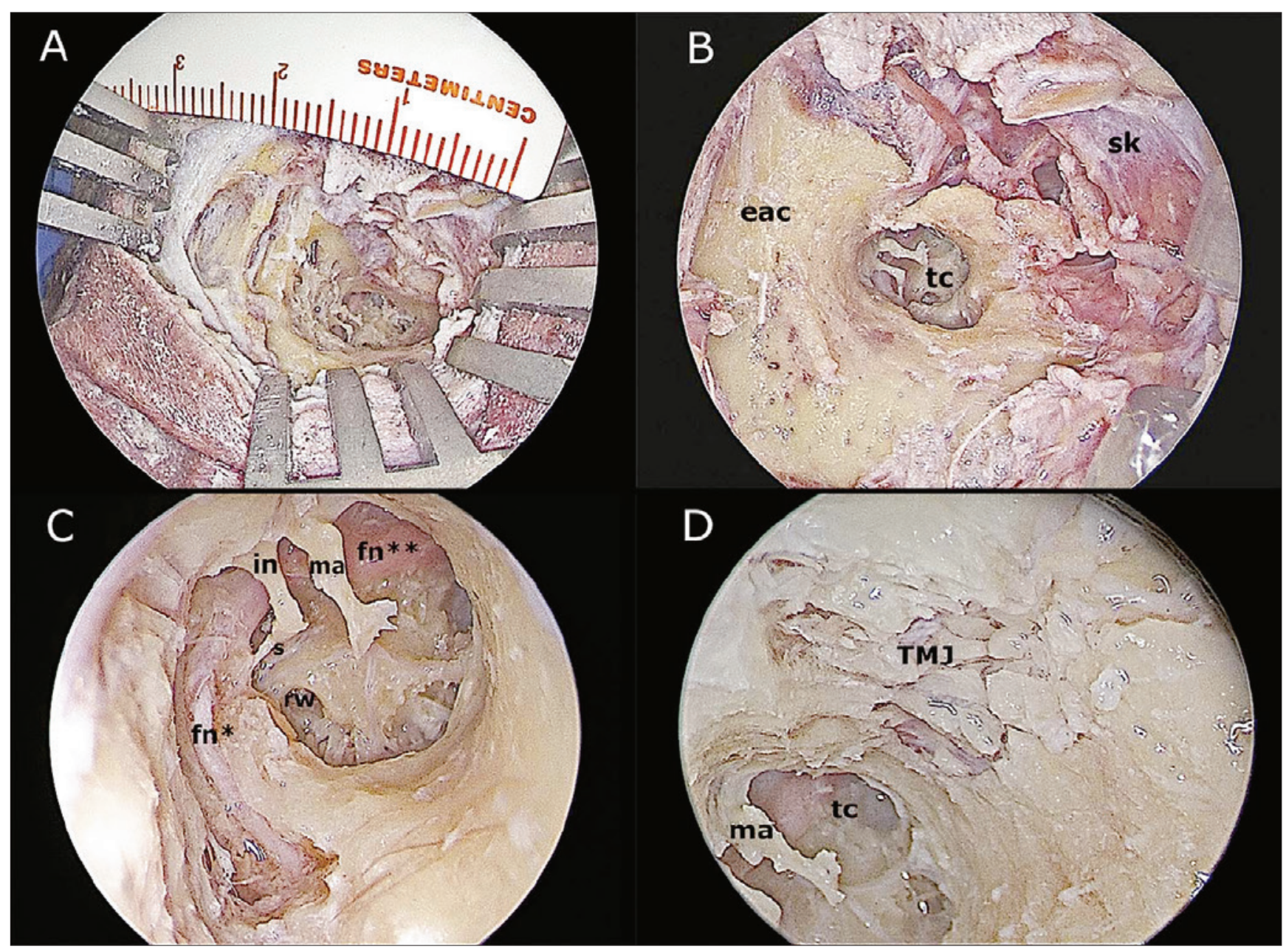

Fig. 1. Shambaugh incision and removal of the tympanic membrane along with the skin of the external auditory canal (A and B); identification of the facial nerve (C) and the temporo-mandibular joint (D). eac, external auditory canal; tc, tympanic cavity; sk, meatal skin; $\mathrm{fn}^{\star}$, third tract of the facial nerve; fn ${ }^{\star \star}$, second tract of the facial nerve; in, incus; ma, malleus; s, stapes; rw, round window niche; TMJ, temporo-mandibular joint. 


\section{Techniques and technologies}

In September 2015, two fresh cadaver heads (4 sides) were dissected using an endoscopic technique by the first author (LP). An expanded approach was codified and named expanded transcanal transpromontorial approach (ExpTTA). Video and photographic material were collected, and a retrospective review and analyses of data obtained by this dissection was performed in October 2015.

\section{Surgical technique}

The head was slightly extended and rotated contra-laterally, just as in the traditional endoscopic middle ear surgery. The surgeon held a $4 \mathrm{~mm}$ diameter, $15 \mathrm{~cm}$ length, $0^{\circ}$ angled endoscope (Karl Storz Tuttlingen Germany) with the left hand, and the operative instruments with the right hand. The endoscope was connected to an AIDA threechip high-resolution monitor and camera system (Karl Storz, Tuttlingen, Germany).
Approach to the tympanic cavity and identification of the main landmarks

The first step was a circular incision of the external ear canal skin approximately $1.5 \mathrm{~cm}$ from the tympanic annulus, under classical traditional endoscopic view, with the endoscope introduced through the external auditory canal (EAC). The skin was then removed "en bloc" with the tympanic membrane. A Shambaugh incision (intercartilaginous skin incision between helix and tragus) was performed to allow the detachment of the lateral portion and the skin of the EAC to expose widely the bony EAC (Fig. 1A). After positioning orthostatic retractors, the EAC was drilled circumferentially to allow a better view of the surgical field and to allow accurate movements of the surgical instruments in the canal (Fig. 1B and 1C).

The next step was the exposition of the temporo-mandibular joint (TMJ) capsule, an important anatomical landmark for this approach representing the superficial anterior limit (Fig. 1D). It was obtained by drilling the anterior

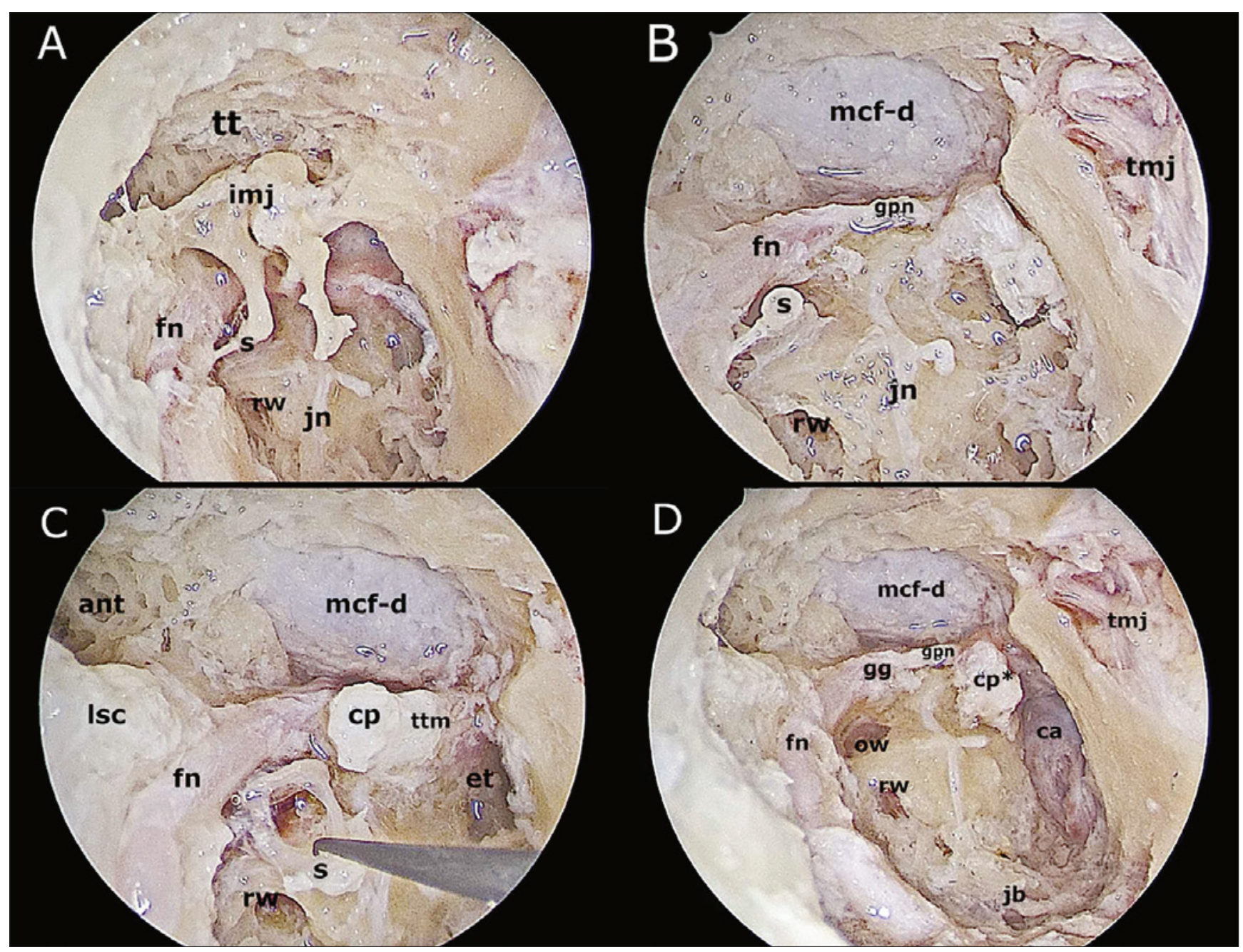

Fig. 2. Removal of the ossicular chain and exposure of the middle cranial fossa dura (A and B); removal of the stapes (C) and skeletonisation of the carotid artery and jugular bulb (D). tt, tegmen tympani; imj, incudo-malleolar joint; fn, facial nerve; s, stapes; rw, round window niche; jn, Jacobson nerve; mcf-d, middle cranial fossa dura; tmj, temporo-mandibular joint; gpn, great petrous nerve; ant, antrum; Isc, lateral semicircular canal; $c p$, cochleariform process; $c p^{\star}$, cochleariform process overturned; ttm, tensor tympani muscle; et, Eustachian tube; ow, oval window; gg, geniculate ganglion; ca, carotid artery; jb, jugular bulb. 
wall of the EAC. A wide atticotomy was made to expose the ossicular chain (Fig. 2A). Consecutively, the incus and the malleus were removed to obtain a clear view of the whole tympanic tract of the facial nerve (Fig. 2B), the geniculate ganglion and its relationship with the cochleariform process. The identification of the main landmarks for this approach continued with the exposition of the middle cranial fossa dura superiorly (by drilling the tympanic tegmen), the carotid artery anteriorly under the tympanic tube orifice (in the protympanic space), the jugular bulb inferiorly and the third tract of the facial nerve posteriorly, drilling the posterior aspect of the EAC and the posterior portion of the bony annulus.

Transpromontorial micro-/endoscopic approach to the IAC

After the clear identification of anatomical landmarks, the dissection proceeded with the removal of the stapes (Fig. 2C) from the oval window and the exposition of the vestibule and the spherical recess in the saccular fossa
(Fig. 2D). This structure appears like a thin cribriform plate separating the vestibule from the fundus of the IAC and represents the site of medial termination of the inferior vestibular nerve fibers.

The enlargement of the oval window was made by a microcurette, a burr or by a Piezosurgery ${ }^{\circledR}$ instrument (Mectron, Carasco/Genova, Italy). At this stage, a transpromontorial approach to the IAC was performed (Fig. 3A), drilling the promontorial bone and exposing progressively the basal, middle and apical turns of the cochlea.

Knowledge of the position of the labyrinthine tract of the facial nerve was allowed by previous identification of all the anatomical structures described that were at the same time boundaries of the surgical field and surgical landmarks. An imaginary line passing from the geniculate ganglion to the spherical recess just above the apical turn of the cochlea indicated the facial nerve route through the inner ear.

The progressive drilling of the IAC was performed until the fundus of the IAC was opened, at the level where the

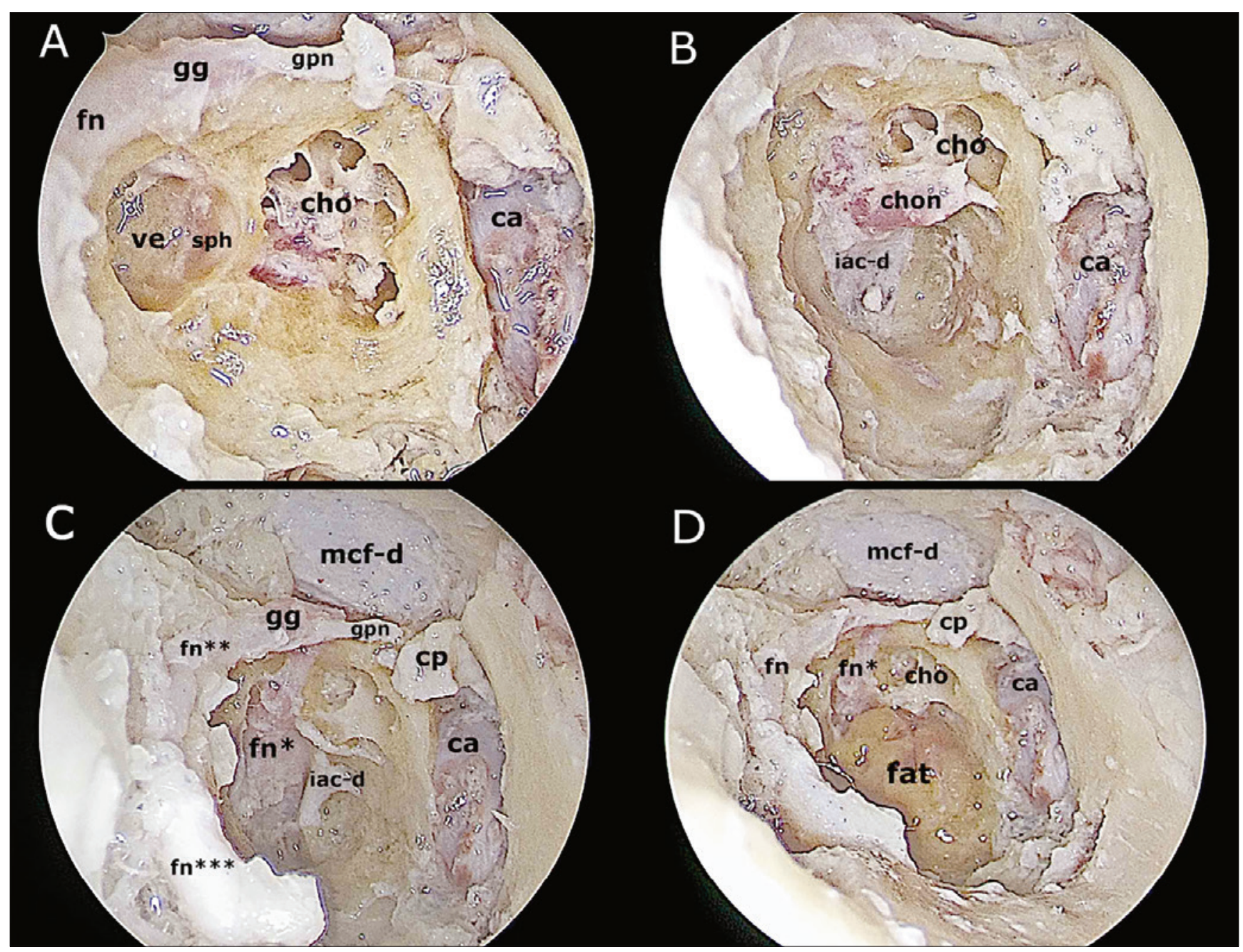

Fig. 3. Approach to the fundus of the IAC and identification of the first tract of the facial nerve. $f n$, facial nerve; fn*, facial nerve first tract; fn**, facial nerve second tract; $\mathrm{fn}^{\star \star \star}$, facial nerve third tract; cho, cochlea; gg, geniculate ganglion; gpn, great petrous nerve; sph, spherical recess; ca, carotid artery; chon cochlear nerve; iac-d, internal auditory canal dura; $c p$, cochleariform process; mcf-d, middle cranial fossa dura; fat, abdominal fat. 
cochlear nerve emerges (Fig. 3B). Our limits of dissection at this point were the second tract of the facial nerve superiorly, the vertical tract of the internal carotid artery anteriorly, the jugular bulb inferiorly, the third portion of the facial nerve posteriorly and the middle cranial fossa dura superiorly (Fig. 3C). The dissection kept on until the lateral aspect of the IAC dura was completely exposed. The dura along the IAC was then cut to reach the internal auditory canal.

The cerebellopontine angle was reached with further bone drilling to enlarge the opening of the IAC meatus, always keeping in mind the anatomical boundaries of the dissection to avoid noble structures injuries, and following the acoustic-facial bundle. Finally, the obliteration of the internal auditory canal was obtained by abdominal fat (Fig. 3D).
In all 4 sides of the cadavers the procedure was feasible, and all the landmarks reported above were identified (Fig. 4). In all cadavers it was necessary to extensively drill the TMJ and to calibrate the EAC to allow adequate room to maneuver the instruments and optics and to comfortably access the CPA. Additionally, the wide skeletonisation of the carotid artery and the jugular bulb were necessary for the same purpose.

\section{Discussion}

Actually, the IAC is a very poor accessible anatomical region despite the different approaches chosen. By a retrosigmoid approach, craniotomy and an extensive drilling of the posterior aspect of the petrous bone are required to fully expose the IAC. In most cases, the use of endo-

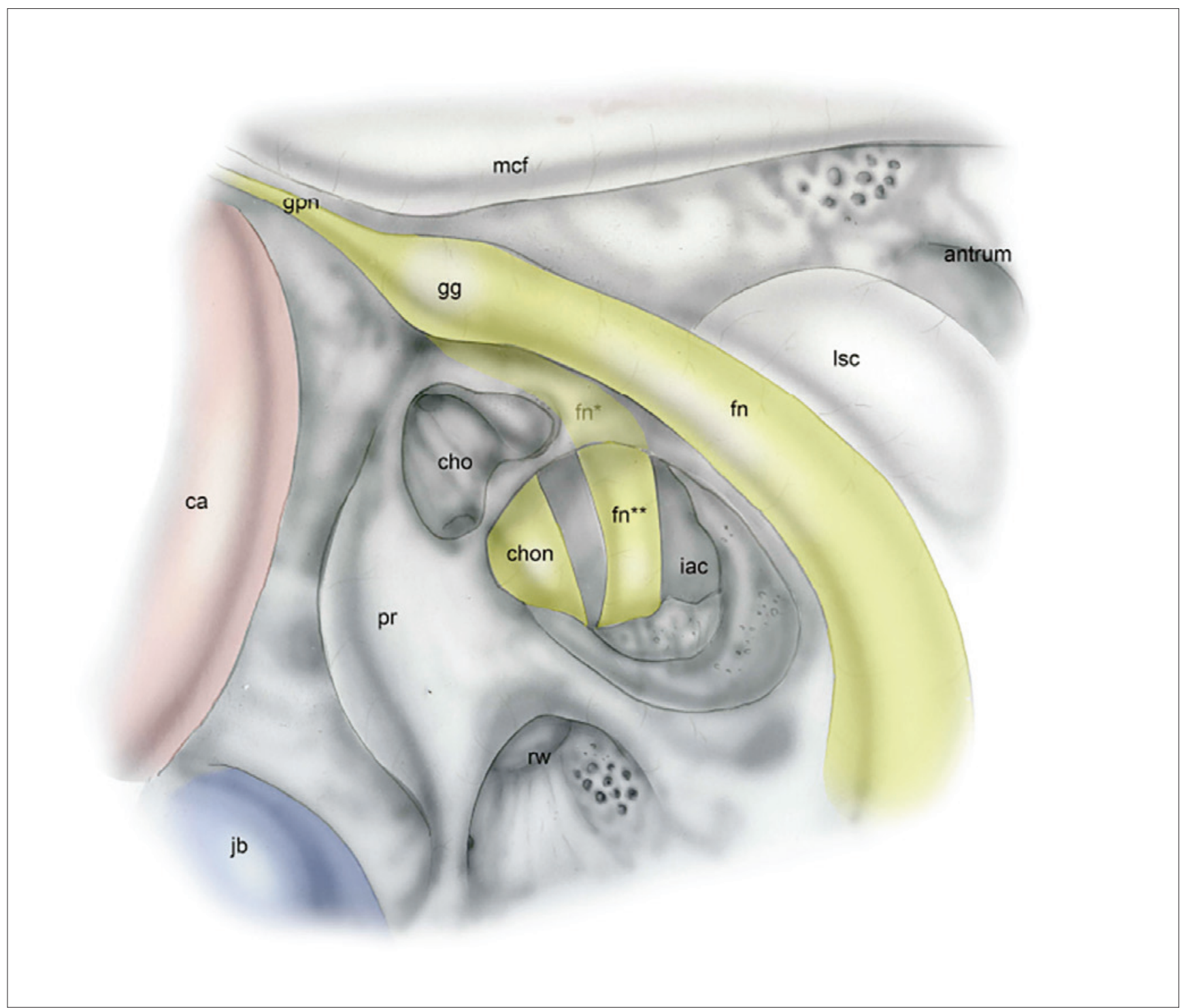

Fig. 4. (Left ear) picture showing the main landmarks of the approach. Jb, jugular bulb; ca, carotid artery; pr, promontory; chon, cochlear nerve; fn, facial nerve; $\mathrm{fn}^{\star}$, intralabyrinthine facial nerve; $\mathrm{fn}^{\star \star}$, facial nerve on the internal auditory canal; iac, internal auditory canal; $\mathrm{rw}$, round window; Isc, lateral semicircular canal; mcf, middle cranial fossa; gg, geniculate ganglion; gpn, greater petrosal nerve. 
scopes inside the CPA is required to visualise the fundus of the IAC. By a translabyrinthine approach, subtotal petrosectomy is required to identify the IAC and to properly skeletonise it. The middle cranial fossa approach guarantees less bone work to the petrous bone, but requires wide craniotomy and temporal lobe retraction ${ }^{1}$. Independently of the approach, the surgery of this region (e.g. VS surgery) is traditionally considered very delicate overall. Post-operative morbidity can be high, due to intraoperative and post-operative complications. Besides this, facial nerve post-operative results are critical for functional and psychological issues and the patient's quality of life. For these reasons, a general attitude in management is to encourage in most cases wait and see policies, so as to evaluate the growth of the mass over time ${ }^{13}$. In case of documented growth, a therapeutic attempt can be more strongly suggested.

In 2013, our team published the first case of EndoTTA ${ }^{10}$. The approach guaranteed cochlear schwannoma removal, with IAC extension. Since this first clinical application, we have started using the approach more frequently for stage I and II (Koos) VSs and in 2015 the first case series of 10 patients was published ${ }^{11}$. The EndoTTA gives the possibility of lateral to medial control of IAC, with a high magnification of every structure inside and outside the IAC, including the facial nerve. The morbidity, based on our first results, can be compared to those of a tympanoplasty, rather than to an operation to the CPA. Certainly, the sample size is still small, since at present the indication to EndoTTA for VS treatment is considered as follows: growing VS stage I or II (Koos), with class D hearing (AAO-HNS) and whose symptoms does not respond to medical treatment (e.g. intratympanic gentamicin injections in case of debilitating vertigo). Nonetheless, considering these very strict indications, we believe that EndoTTA is very promising, since it potentially differs in terms of morbidity from classic microscopic approaches. Moreover, it guarantees radical removal of the pathology, with a possible very low morbidity to the facial nerve, due to direct control and magnification of the entire nerve path thanks to the endoscope. Of course, hearing preservation is not feasible by this approach, and it is for this reason that the indication to surgery is restricted to patients with unserviceable hearing.

ExpTTA, as shown herein, may potentially expand the anatomical limits of the indication to surgery for two main reasons. The first is an obviously enlarged space for maneuvering surgical instruments, compared to EndoTTA. The second one is, as a direct consequence of the increased space for surgical instruments, the possible use of a microscope in combination with the endoscope for some delicate steps, for example while dissecting vessels in the most medial portion of the pathology, or towards the CPA. The use of a microscope would free one hand during the dissection, facilitating the procedure when necessary.

Morbidity, although this needs to be confirmed in living patients, would be theoretically similar to the EndoTTA, since it involves only a small skin incision between tragus and helix (Shambaugh incision), and only a small increase in bone work.

In summary, this approach can be considered a sort of lessinvasive translabyrinthine approach, since it demolishes the labyrinth, but it spares the mastoid, most of the temporal bone and avoids large skin incisions and wide soft tissue dissections. Of course, clinical experience is necessary to confirm its potential benefits and define the feasibility and morbidity of this expanded approach. The risks of the approach must also be highlighted: actually, going medially toward CPA the risk of incontrollable bleeding, possibly from branches of the anterior inferior cerebellar artery (AICA) would increase, and the room created would not be enough to control it. Moreover, drilling of the internal carotid artery could be theoretically associated with a potential risk of carotid artery injury, and drilling the temporomandibular joint could lead to a potential discomfort to the patient. Moreover, although EndoTTA has very low risk of complications such as post-operative cerebrospinal fluid leak, or facial nerve palsy, the ExpTTA could have potential higher rates of unfavourable events. Also, the small number of specimens dissected does not take in consideration the chance of some anatomical variations such as a high jugular bulb, a more medial internal carotid canal in the temporal bone, a temporo-mandibular joint protrusion in the external auditory canal, a very low middle cranial fossa dura tegmen and lastly a more medial course of the third portion of the facial nerve. All these anatomical variations lead to very limited exposure of the surgical area and consequently of the operative field in the CPA by ExpTTA. Finally, all types of endoscopic lateral skull base procedures require preliminary, long training in endoscopic middle ear surgery to acquire enough manual expertise. Additionally, perfect knowledge of endoscopic landmarks is necessary to recognise and dissect in the safest way the neurovascular structures inside the temporal bone.

\section{Conclusions}

ExpTTA is a feasible approach to access the IAC and cerebellopontine area. Potential extensive and routine application of this approach in lateral and posterior skull base surgery will depend on the development of technology and surgical refinements, and on the diffusion of skull base endoscopic skills among otolaryngologists and the neurosurgical community.

\section{Acknowledgements}

LA acknowledges a research fellowship by the Bangerter-Rhyner Foundation, Bern, Switzerland and Karl Storz $\mathrm{GmbH}$, Tuttlingen, Germany. The funders had no role in study design, data collection and analysis, decision to publish or preparation of the manuscript. 


\section{References}

1 Bennett M, Haynes DS. Surgical approaches and complications in the removal of vestibular schwannomas. Otolaryngol Clin North Am 2007;40:589-609.

2 Magnan J, Chays A, Lepetre C, et al. Surgical perspectives of endoscopy of the cerebellopontine angle. Am J Otol 1994;15:366-70.

3 Thomassin JM, Korchia D, Doris JM. Endoscopic guided otosurgery in the prevention of residual cholesteatomas. Laryngoscope 1993;103:939-43.

4 Presutti L, Marchioni D, Mattioli F, et al. Endoscopic management of acquired cholesteatoma: our experience. J Otolaryngol Head Neck Surg 2008;37:481-7.

5 Tarabichi M. Endoscopic management of limited attic cholesteatoma. Laryngoscope 2004;114:1157-62.

6 Marchioni D, Alicandri-Ciufelli M, Molteni G, et al. Endoscopic tympanoplasty in patients with attic retraction pockets. Laryngoscope 2010;120:1847-55.

7 Marchioni D, Alicandri-Ciufelli M, Rubini A, et al. Endoscopic transcanal corridors to the lateral skull base: Initial experiences. Laryngoscope 2015;125 Suppl 5:S1-13.
8 Presutti L, Nogueira JF, Alicandri-Ciufelli M, et al. Beyond the middle ear: endoscopic surgical anatomy and approaches to inner ear and lateral skull base. Otolaryngol Clin North Am 2013;46:189-200.

9 Marchioni D, Alicandri-Ciufelli M, Mattioli F, et al. From external to internal auditory canal: surgical anatomy by an exclusive endoscopic approach. Eur Arch Otorhinolaryngol 2013;270:1267-75.

10 Presutti L, Alicandri-Ciufelli M, Cigarini E, et al. Cochlear schwannoma removed through the external auditory canal by a transcanal exclusive endoscopic technique. Laryngoscope 2013;123:2862-7.

11 Marchioni D, Alicandri-Ciufelli M, Rubini A, et al. Exclusive endoscopic transcanal transpromontorial approach: a new perspective for internal auditory canal vestibular schwannoma treatment. J Neurosurg 2016;11:1-8.

12 Thakur JD, Banerjee AD, Khan IS, et al. An update on unilateral sporadic small vestibular schwannoma. Neurosurg Focus 2012;33:E1.

13 Patnaik U, Prasad SC, Tutar H, et al. The long-term outcomes of wait-and-scan and the role of radiotherapy in the management of vestibular schwannomas. Otol Neurotol 2014;36:638-46.

Received: May 31, 2016 - Accepted: November 23, 2016

Address for correspondence: Lukas Anschuetz, Department of Otorhinolaryngology, Head \& Neck Surgery, University Hospital of Modena, via del Pozzo 71, 41100 Modena, Italy. Tel. +390594 222402. Fax +39 0594 222454. E-mail: anschuetz.lukas@ gmail.com 